\title{
Los desafíos de la educación superior en el contexto de la globalización*
}

\author{
José Solano Alpizar \\ María Ester Aguilar Mora
}

\begin{abstract}
Resumen: La presente ponencia realiza una caracterización de algunos de los desafios que enfrenta la educación superior frente al acelerado proceso de cambios que ha propiciado la globalización, y sugiere posibles cursos de acción que contribuyan a que la educación superior se adecúe al contexto sin perder su compromiso socio-cultural con la sociedad costarricense.

Para efectos expositivos la ponencia se estructura en tres partes: en la primera se realiza una breve caracterización del fenómeno de la globalización, en la segunda se precisan algunos de los desafios a los que se enfrenta la educación superior como producto de las transformaciones de su entorno, tanto nacional como internacional, y en la tercera parte se enuncian algunos retos que requieren de un proceso de reflexión permanente por parte de las personas que se encuentran comprometidas con la educación superior.
\end{abstract}

"Cualquier institución que duplica o triplica su tamaño, o que sobreviva tres décadas, tiene que reinventarse a sí misma"

Drucker

\section{Algunas consideraciones sobre la globalización: a manera introductoria}

Los procesos de internacionalización de la producción, los mercados y las finanzas han propiciado un orden de relaciones entre los países y los propios actores sociales que no tiene precedentes en el ámbito histórico. Nunca antes el mundo había experimentado un proceso a escala planetaria cuya envergadura se encuentra modificando no sólo la realidad interna de los países sino también su inserción en el mercado internacional (Ferrer, 1996, p. 1367). Este proceso conocido como "globalización" ha trascendido las fronteras nacionales impactando los más diversos espacios societales en sus dimensiones, económico, político, social y cultural.

En el ámbito económico se expresa como un proceso que ha permitido el surgimiento de redes y sistemas internacionales de producción y comercialización, el que se caracteriza por la velocidad y dinamismo de 
los cambios y las innovaciones científico-tecnológicas así como por la velocidad de propagación y creciente simultaneidad e impacto de los flujos de capital, bienes e ideas, a lo que debemos de sumar la mayor especialización de los circuitos de comunicación.

En un plano político y social podemos decir que la globalización ha representado un lugar de encuentro para todo un conjunto de procesos que van desde la democratización de nuestras sociedades -en términos de una mayor participación de la sociedad civil en los asuntos de la vida pública que los afectan directa e indirectamente- hasta la búsqueda de la equidad en materia económica, política y social y la transformación de las instituciones en términos de su estructura y sus funciones; pero también ha generado una mayor exclusión social reflejada en el incremento de la pobreza, la marginación de amplios sectores de los frutos del crecimiento económico, la transferencia de tareas del sector público al privado y los cambios en las relaciones de producción y pautas de comportamiento de amplios sectores sociales, por mencionar algunos.

En el ámbito cultural este proceso se expresa en el pasaje de identidades culturales tradicionales y modernas de base territorial a otras modernas y postmodernas de carácter transterritorial que no se estructuran desde la lógica de los estados-naciones sino desde la lógica de los mercados; razón por la que no se basan -en lo esencial- en comunicaciones orales y escritas sino que funcionan por medio de la producción industrial de la cultura, su comunicación tecnológica y el consumo diferido y segmentado de los bienes.

Como tal, la globalización no puede ser comprendida al margen de la revolución científico-técnica que ha venido experimentando el mundo contemporáneo. Revolución que se ha venido extendiendo sobre la base de la difusión de las tecnologías derivadas de la microelectrónica, en especial las vinculadas al procesamiento y transmisión de información (CEPAL, 1992), lo que ha generado un cambio radical en las formas de producción y de consumo, pues la sofisticación de los equipos electrónicos y las redes de comunicación han propiciado nuevas prácticas comerciales y financieras así como nuevas relaciones en el ámbito de la producción y el consumo.

Contrario a anteriores revoluciones, ésta revolución se caracteriza por la articulación cada vez más estrecha entre el desarrollo científico, los avances tecnológicos y su aplicación en la esfera de la producción, la distribución y el consumo de bienes y servicios, lo cual sugiere la imagen de un proceso productivo distinto del que conocíamos hasta hace un par de décadas, pues hoy en día el proceso de producción se caracteriza por la fragmentación geográfica y la especialización en manufacturas de piezas o de componentes en función de ventajas comparativas de fabricación así como por los cambios en los patrones de consumo que están tendiendo a ser menos diferenciados debido al creciente poder de los medios de comunicación (CEPAL, 1987, chesnais, 1992).

Este entorno productivo cambiante ha propiciado la emergencia de una propuesta de desarrollo en América Latina afirmada en la necesidad de una transformación productiva que se caracteriza por la exigencia de elevar los niveles de competitividad y equidad ${ }^{1}$. En ella se plantea la necesidad de que los países de la región -respetando su especificidad- inicien un proceso de modernización a través de una nueva fase de industrialización -menos sectorial y más entrelazada con la agricultura y los servicios- sobre la base de la incorporación del progreso técnico (CEPAL, 1990).

Esta exigencia de incorporación y difusión del progreso técnico (CEPAL/UNES$\mathrm{CO}, 1992)$ ha venido generando que el ciclo 
de acumulación del capital dependa cada vez menos de la intensidad de los recursos naturales y del trabajo -incluso de la intensidad de capital productivo- y cada vez más de la acumulación tecnológica basada en la intensidad de conocimiento.

Dicha acumulación de conocimientos supone una complementación entre creación, innovación y difusión; razón por la cual se hace imprescindible la adopción de ciertos procesos de aprendizaje tales como los de -learning by doing- aprendizaje mediante la práctica, -learning by usingaprendizaje mediante el uso de sistemas complejos y -learning by interacting- interacción entre productores y consumidores (CEPAL/UNESCO, 1992).

En este sentido debemos decir que la globalización ha propiciado que el conocimiento se convierta en el principal factor de producción, pues tal y como lo apuntan Toffler (1990) y Drucker (1992) en el nuevo ordenamiento económico mundial la forma de alcanzar el desarrollo y el poder económico no está dado por la explotación de las materias primas y del trabajo del hombre sino por él conocimiento.

Esta importancia atribuida al conocimiento en el contexto de la globalización supone no sólo la redefinición del papel de la educación de entrada al siglo XXI sino que exige un replanteamiento de la educación superior para afrontar los desafíos y los retos del nuevo entorno económico-social.

\section{Los desafíos de la educación superior en el contexto de la globalización}

En América Latina la aceptación de la globalización coincidió con el cuestionamiento generalizado que se comenzó a hacer de la educación superior a finales de la década del ochenta e inicios de la del noventa.
La denominada "crisis de la educación superior” se evidenció aún más, cuando la educación superior comenzó a ocupar un espacio de discusión importante en el mundo intelectual y en la agenda de diversos organismos internacionales (Brunner, 1992; Kent, 1992; Schwartzman, 1993; Banco Mundial, 1994; UNESCO, 1995, 1998).

Los cuestionamientos acerca de su pertinencia y su calidad afloraron en el momento en que los teóricos del desarrollo reconocieron los nexos existentes entre los retos competitivos de nuestras economías y la eficacia del sistema educativo, pues una vez más, las relaciones entre educación y desarrollo emergían como puntales de una añeja discusión que le atribuye a la educación el papel de pivote para el desarrollo, y le reconoce el ser uno de los principales factores generadores de riqueza en el mundo globalizado.

Este reposicionamiento de la educación y del conocimiento como ejes del desarrollo ha generado fuertes exigencias de formación y cualificación de los recursos humanos que han traído consigo la redefinición de la oferta universitaria y por ende un replanteamiento de sus funciones y de su estructura para responder a los requerimientos del mundo productivo.

\subsection{Dos posiciones en el debate internacional: El Banco Mundial y la UNESCO}

La década del noventa se encuentra marcada por la presencia de un debate sobre la educación superior del cual han sido protagonistas el Banco Mundial y la UNESCO. En el documento del Banco Mundial (1994) Higher education. Lessons from experience $^{2}$ se plantea lo que se considera los problemas críticos de la educación superior; resaltándose como problemas principales: la mala calidad de la educación superior; los 
problemas de eficiencia en el uso institucional de los recursos y los problemas de equidad, entre otros.

Los especialistas han coincidido en aceptar que en el enfoque del banco mundial “... subyace y predomina un supuesto económico: la educación superior es significativa en tanto que valor agregado; es decir en la medida en que regresa al individuo su inversión y en la medida en que favorece la economía nacional y apoya sus opciones de desarrollo...” (Rodríguez, 2000).

De manera tal que resurge con denodada fuerza la visión de "capital humano" que prevalecía en las políticas de la década del sesenta y del setenta, en las que se establecía una correlación positiva entre escolaridad, productividad y desempeño, y donde la educación se constituye en una inversión como cualquier otra.

Frente a esta postura economicista, emerge el enfoque de la UNESCO que sienta algunas de sus posiciones en el documento de la Conferencia Mundial sobre Educación Superior que tuvo lugar en París (1998) y que recoge muchos de los planteamientos desarrollados en toda una serie de documentos y eventos internacionales sobre educación superior que tuvieron lugar a lo largo de la década del noventa.

Se plantea que la educación superior tiene como misión insoslayable servir a la persona humana y a la sociedad a través de su función de investigación, sus cursos de estudio y entrenamiento, sus actividades cooperativas y sus alianzas con varios sectores sociales.

Con esa misión como corolario se plantea que la educación superior debe participar activamente en la solución de los principales problemas globales, regionales y locales (pobreza, hambre, analfabetismo, exclusión social, etc.) y trabajar en la generación de propuestas y recomendaciones que promuevan el desarrollo humano sus- tentable, la ampliación del conocimiento, el respeto universal por los derechos humanos y la igualdad de derechos para mujeres y hombres, entre otros (Rodríguez, 2000).

Este enfoque de la UNESCO es el producto de un consenso amplio que recoge los puntos de vista de una gran variedad y diversidad de actores de la educación. En él, se establece un equilibrio entre los requerimientos del mundo globalizado y la misión tradicional y permanente de la universidad. De suerte tal, que frente a un enfoque que enfatiza los aspectos económicos y de rentabilidad de la educación y la capacitación, encontramos otro que rescata lo político, lo social y lo cultural de la educación que permiten percibir las necesidades de reforma educativa desde otra perspectiva.

\subsubsection{Un enfoque alternativo}

No hizo falta mucho tiempo para que el Banco Mundial y la UNESCO realizaran un trabajo conjunto en el que los aspectos económicos y los aspectos socio-culturales fueran retomados en un mismo documento.

\section{En el documento Higher Educa-} tion in Developing Countries: Peril and Promise (2000) se recogen los aspectos más importantes que han sido planteados en las diferentes actividades sobre educación superior y particularmente como seguimiento de los resultados de la Conferencia Mundial de París en 1998.

En él se analizan los viejos problemas de la educación superior a la luz del nuevo contexto histórico; el papel de la educación superior en el desarrollo económico, social, político y cultural de los países y su interés público; la gestión universitaria; la ciencia y la tecnología y la relevancia de la educación general que no se debe limitar a la tradicional formación especializada. 
De esta manera un nuevo enfoque emerge marcando el derrotero del debate, que sin limitarlo, pone en evidencia las principales líneas de acción por donde debe discurrir el debate nacional y las propuestas de reforma en materia de educación superior.

\section{Los principales desafíos de la educación superior}

Tomando lo anterior como referente, consideramos que algunos de los principales desafíos a los que se enfrenta la educación superior costarricense son: a) reinventarse en el nuevo entorno económico-social, b) relaciones universidad-sociedad, c) adaptación a las exigencias del desarrollo científico-técnico, ch) despliegue de la investigación en una sociedad del conocimiento.

\section{a) Reinventarse en el nuevo entorno económico-social}

La educación superior en América Latina ha quedado en deuda con la sociedad que la vio nacer, pues como bien lo ha dejado asentado García-Guadilla (1988) la distancia entre lo que se esperaba que ella diera y lo que realmente dio, es significativa; sobre todo sí observamos que se esperaba que su expansión ofreciera una distribución justa de los conocimientos y de los recursos, y en realidad generó cierta "homogeneidad en la calidad", fortaleció a aquellas instituciones y disciplinas de mayor calidad y reconocimiento social y promovió una flagrante injusticia distributiva.

Tampoco cumplió con la expectativa de formar profesionales que respondieran a las necesidades del desarrollo, pues la expansión, lejos de producir un contingente de profesionales preocupados por el desarrollo del país, generó, en la mayoría de los casos, un profesional preocupado por hacer rentable su capital cultural, institucionalizado y valorado a través del título universitario.

Peor aún sí observamos que, lejos de ampliar y diversificar espacios de conocimiento lo que se hizo fue "más de lo mismo", pues siendo consecuente con el modelo de desarrollo adoptado en la región a partir de la década del cincuenta, se volvió hacia la producción de los países "industrializados" más para copiar e imitar que para crear o innovar.

A lo anterior debemos sumar lo volátil de las fronteras entre lo público y lo privado que se han convertido en un problema medular para la educación superior en el actual entorno económico-social, pues en un momento de transformaciones profundas en las formas de producir, de redefinición de las funciones del estado y de creciente participación de la sociedad civil en la vida económica, social y política tenemos que repensar las categorías de lo público y lo privado de cara a la creación de nuevas alternativas de financiamiento, al establecimiento de nuevas relaciones con los sectores productivos, y a la formalización de alianzas estratégicas con el mundo empresarial y el estado, entre otros; todo ello sin perder de vista el histórico compromiso social.

\section{b) Relaciones universidad / sociedad}

La universidad debe reinventarse también en cuanto a sus relaciones con la sociedad en el mundo globalizado; pues el nuevo entorno económico-social exige contribuir al fortalecimiento de la democracia a través de un ejercicio equitativo en la distribución de los conocimientos y los recursos. De igual manera que comprometiéndose a contribuir en la satisfacción de 
la necesidades básicas de la población por medio de programas específicos y mediante nuevas formas de capacitación que atiendan las necesidades más inmediatas de la población, sobre todo de aquella que se encuentra marginada de los frutos del crecimiento económico por la propia dinámica impuesta por la globalización.

Siendo consecuente con lo planteado en la conferencia sobre educación superior en París (1998), la universidad debe comprometerse en el combate de la pobreza y de la creciente exclusión social, promoviendo programas formales, no formales e informales que atiendan problemas concretos como el de analfabetismo, la violencia doméstica y la drogadicción; y atender el ámbito de los derechos humanos, la participación ciudadana, el desarrollo sustentable y la igualdad de derechos entre hombres y mujeres, entre otros.

$\mathrm{Su}$ vinculación con la tecnología y la formación de los recursos que requiere el mundo productivo no deben convertirse en óbice para que pierda su perspectiva humanista, que en un mundo de competitividad e individualismo, requiere de una buena dosis de humanización del "capital humano", pues se requiere no perder de vista la necesidad de una universidad más vinculada con los distintos sectores sociales.

\section{c) Adaptación a las exigencias del desarrollo científico-técnico}

El mundo globalizado -tal y como se señaló en la parte introductoria- ha convertido el conocimiento en eje de la transformación productiva. La educación superior tiene que asumir como uno de sus principales desafíos la adaptación a las exigencias del desarrollo tecnológico y científico para poder insertarse en la nueva sociedad del conocimiento.
Adaptación que pasa por el cambio en las formas de gestión, las modalidades de organización del trabajo, el rediseño y la renovación curricular, la eficiencia y la evaluación permanente, el ensayo de modalidades alternativas de formación, la flexibilización, el intercambio y la movilidad inter-universitaria de graduandos, docentes e investigadores y la formación de formadores, entre otros. Además se requiere que la educación contemple, no ya la imitación y la copia, sino el desarrollo de verdaderos procesos de creación, producción e innovación de conocimientos. Para ello se debe promover la investigación, dotando de recursos a los investigadores y generando mayor trabajo interdisciplinario, tanto al interior de una universidad como entre estas; y por supuesto a nivel internacional.

Se tiene que apostar a la educación continua, reconociendo que la formación no termina en un ciclo determinado sino que se mantiene a lo largo de la vida, y por ello se debe ofrecer las posibilidades para que la gente pueda formarse e informarse en un mundo en el que los conocimientos quedan obsoletos en cuestión de una década.

Los espacios para la actualización permanente del profesional resultan imprescindibles para que las personas, en cualquier etapa de su vida, puedan reincorporarse a la vida académica y mantenerse actualizados.

Esta adaptación también pasa por el reconocimiento de una mayor y mejor vinculación con el mundo productivo y el mercado de trabajo. En este contexto es deseable que la educación superior pueda -además- ofrecer respuestas de calificación a los trabajadores, es decir, que ofrezca la posibilidad de un reciclaje laboral para aquellos grupos y sectores que se encuentran en desventaja respecto a las innovaciones en su campo de trabajo. 


\section{ch) Despliegue de la investigación en la sociedad del conocimiento}

De acuerdo García-Guadilla (1988) en el debate contemporáneo sobre la educación superior se baten diversas posiciones respecto del papel de la investigación en la universidad. La primera considera que se debe defender la calidad de la educación superior pública y desarrollar la investigación; la segunda sostiene que se debe preservar el espacio público pero dividido en dos niveles: el de la excelencia (donde se haría investigación) y el masificado (de transmisión de conocimientos); y la tercera afirma que la investigación debería realizarse en instituciones diferentes a las universidades ${ }^{4}$.

En el primer caso, los defensores consideran que no es posible desvincular docencia de investigación. Allí producción y docencia se tocan en sus extremos y dan vida a esta posición teórica.

Los segundos parten del supuesto de que deben existir dos niveles, uno de carácter selectivo en el que quiénes ingresan deben tener una formación de alto nivel y otro de carácter más masificado. En el primer nivel existiría una visión eficientista según la cual se contrataría un plantel básico de investigadores de tiempo completo con remuneraciones muy por encima del promedio y de gran experiencia en el terreno académico y el de la investigación.

La tercera posición propone que las universidades queden como transmisoras de conocimientos y que la producción de conocimientos (investigación) se haga en redes extrauniversitarias. Para este grupo la investigación requiere un creciente grado de especialización y profesionalización que deviene incompatible con las exigencias por una docencia cada vez más masiva.

En Costa Rica estas posiciones se expresan de muy diversa manera, es así co- mo podemos encontrar quienes creen oportuno separar la docencia de la investigación y por ello recluyen a la investigación en los centros e institutos, divorciando el saber sabio del saber enseñado. Otros creen que investigación y docencia deben ir de la mano, por cuanto no se puede enseñar algo sí este no ha sido investigado. Un tercer grupo cree que la universidad es un espacio para impartir docencia (un gran enseñadero) y que la investigación no sobra pero tampoco falta.

\section{Consideraciones finales: puntos de partida y puntos de llegada}

- $\quad$ En Costa Rica se necesita fortalecer el proceso de investigación en el ámbito de la Educación Superior, de manera que nos posibilite obtener una fotografía en retrospectiva de lo que ha sido la educación superior y su proceso de desarrollo. Un análisis en ésta dirección nos podría ofrecer muchas luces acerca de la dirección que se debería tomar en aspectos básicos para una reforma educativa integral.

- $\quad$ Es necesario establecer con claridad y precisión las metas que dirigen el accionar de la universidad al cumplimiento de sus compromisos con el desarrollo económico y la democratización de la sociedad, para evitar que se desvié completamente de su misión tradicional y permanente.

- Desarrollar formas creativas de financiamiento de la educación superior sin dejar de lado la importancia del estado y su obligado compromiso financiero para con las casas de enseñanza superior.

- Replanteamiento de la educación superior a partir de cambios en el 
mundo del trabajo que exigen transformaciones significativas en materia disciplinar, reconociendo la necesidad de revisar contenidos, métodos y medios de enseñanza, de manera que se propicie en el estudiante el aprender para emprender, con ello el profesional se prepara no sólo para ser empleado sino también para crear unidades productivas $\mathrm{y}$ generar empleo.

- $\quad$ Formación de formadores es un tema insoslayable en la discusión, pues requerimos dar respuestas acordes con los tiempos, ya que nos encontramos en muchos casos, con procesos de formación y con personal formado desde una perspectiva tradicionalista y conservadora a pesar de que se maneja un discurso modernizante.

- La universidad debe contribuir al desarrollo de la conciencia crítica de la sociedad para hacer posible la propuesta de alternativas que permitan enfrentar una realidad cambiante y dinámica, de lo contrario se seguirán presentando realidades ontologizadas como las únicas vías para acceder al desarrollo -vg. el fenómeno de la globalización-.

- $\quad$ Para concluir es preciso señalar la necesidad de que la educación superior dentro del marco de los cambios que se están dando, asuma el reto de ser instrumento para contribuir en el desarrollo de una sociedad más justa, abierta y solidaria.

\section{Notas}

* La presente ponencia retoma en la parte introductoria, aspectos de un texto previo de los autores: (2000) "La educación rural en el contexto de la globalización”. Educación Rural.
Un acercamiento pedagógico. Centro de Investigación y Docencia en Educación, División de Educación Rural, Universidad Nacional de Costa Rica. Heredia, Costa Rica: Imprenta Segura Hermanos.

1. Nos referimos a la propuesta presentada por la CEPAL en el año de 1990 bajo el título de "Transformación productiva con equidad: la tarea prioritaria de América Latina y el Caribe”. Santiago: CEPAL.

2. Decimos "una vez más" por qué existe suficiente documentación acerca de esta relación en la literatura del periodo anterior a los ochentas. Al respecto véase José Solano (2001) Educación y Desarrollo en América Latina: un análisis histórico-conceptual. EUNA: Heredia.

3. Tomando como base los resultados de un estudio en el África subsahariana, el Banco Mundial realiza una extrapolación y generaliza sus conclusiones y recomendaciones al resto de "países en desarrollo" sin más ni más.

4. En nuestro país esta discusión no ha alcanzado todavía el vuelo que se requiere, pues a pesar de que podemos encontrar posiciones al respecto, estas se presentan de manera aislada e inconexa y no tanto como fruto de un ejercicio colegiado e institucional.

\section{Referencias bibliográficas}

Aguilar Mora, María Ester, Solano Alpízar, José et al. Educación Rural. Un acercamiento pedagógico. Centro de Investigación y Docencia en Educación, División de Educación Rural, Universidad Nacional de Costa Rica. Heredia, Costa Rica: Imprenta Segura Hermanos. 2000.

World Bank. Higher Education. Lessons from Experience. Washington, DC: World Bank. 1994.

CEPAL. "El desarrollo de América Latina y el Caribe: Escollos, requisitos y opciones". En: Cuadernos de la CEPAL. Santiago: CEPAL. 1987. 
Transformación productiva con equidad: La tarea prioritaria de América Latina y el Caribe. Santiago: CEPAL. 1990.

CEPAL/UNESCO. Educación y conocimiento: Eje de la transformación productiva con equidad. Santiago: CEPAL. 1992.

Chesnais, Francois. "La globalización y el estado del capitalismo a finales de siglo". En: Investigación económica, núm 215, México. 1996.

Drucker, Peter. Las nuevas realidades: en el estado y la política... en la economía y los negocios... en la sociedad y en la imagen del mundo. Buenos Aires: Editorial Sudamericana. 1992.

Ferrer, Aldo. "Desarrollo y subdesarrollo en un mundo global: los problemas de América Latina”. En: El trimestre económico. Vol. LXIII (4), Núm. 252, México. 1996.

García-Guadilla, Carmen. "Educación superior en América Latina: desafíos conceptuales, dilemas y algunas proposiciones temáticas para la década del noventa”. En: Revista Paraguaya de Sociología. Año 25, núm. 73, setdic. Asunción. 1988.
Kent, Rollin. Expansión y diferenciación del sistema de educación superior en México, 1960 a 1990. En: Cuaderno de Investigación, núm. 21. Departamento de Investigaciones Educativas, CINVESTAV, Instituto Politécnico, México: DIE. 1992.

Navarro, Juan Carlos. "Una agenda de investigación en educación superior para América Latina: explorando las consecuencias de diversas perspectivas disciplinarias". En: Sociológica, año 13, núm. 36, enero-abril. Universidad Autónoma Metropolitana. México. 1998.

Rodríguez, Roberto. "La reforma de la educación superior. Señas del debate internacional de fin de siglo". En: Trayectorias. Revista de Ciencias Sociales. Universidad Autónoma de Nuevo León, año 2, núm. 3, mayo-agosto. 2000 .

Toffler, Alvin. El camino del poder. Barcelona: Plaza y Janés Editores. 1990.

Schwartzman, Simon. "Policies for Higher Education in Latin America: the context", Higher Education. Vol 25, núm. 1, January. 1993. 